\title{
NOTE ON A CERTAIN TYPE OF DIOPHANTINE SYSTEM ${ }^{1}$
}

\author{
E. T. BELL
}

1. The quadratic case. Several writers ${ }^{2}$ have considered the problem of making $a^{2} x^{2}+d x, b^{2} x^{2}+e x, \cdots$ simultaneously squares, $a, d$, $b, e, \cdots$ being constants, without discussing the conditions under which an integer solution exists. The fact that the coefficients of $x^{2}$ are squares is of no consequence. It is shown in $\$ 2$ how necessary and sufficient conditions for the existence of integer solutions in much more general problems $(\$ 2,(9),(13))$ can be determined and how, when these conditions are satisfied, the solutions may be found. The details are given first for three very special cases, (1), (5), (6) below.

If all letters denote integers, and $a, b, c, d$ are constant, we seek necessary and sufficient conditions that the system

$$
a x^{2}+b x=y^{2}, \quad c x^{2}+d x=z^{2}
$$

shall have a solution $x, y, z$. We shall assume that $a b c d \neq 0$, as the excluded possibilities require only slight modifications, all of which are included in the general method.

The required conditions are that $b, d$ be simultaneously representable in two forms of degree seven. Precisely, (1) has a solution in integers $x, y, z$ if and only if integers $f, g, h, k, m, n$ exist such that

$$
b=h f^{2}\left(m^{2}-a g^{2} k^{2}\right), \quad d=h g^{2}\left(n^{2}-c f^{2} k^{2}\right) .
$$

Provided such $f, g, \cdots$ exist, the complete solution of (1) is

$$
x=h f^{2} g^{2} k^{2}, \quad y=h f^{2} g k m, \quad z=h f g^{2} k n,
$$

where $f, g, h, k, m, n$ run through all solutions of (2).

To prove this, we rewrite (1) as

$$
x(a x+b)=y^{2}, \quad x(c x+d)=z^{2},
$$

which is of the form

$$
x u=y^{2}, \quad x v=z^{2},
$$

with

$$
u \equiv a x+b, \quad v \equiv c x+d .
$$

The complete integer solutions of the respective equations in (3),

${ }^{1}$ Presented to the Society, April 6, 1940.

${ }^{2}$ Summary of results to 1920 in L. E. Dickson, History of the Theory of Numbers, vol. 2, 1920, chap. 18. 
with $x, y, z, u, v$ unrestricted variables, are

$$
\begin{array}{lll}
x=r_{1} s_{1}^{2}, & u=r_{1} t_{1}^{2}, & y=r_{1} s_{1} t_{1} ; \\
x=r_{2} s_{2}, & v=r_{2} t_{2}^{2}, & z=r_{2} s_{2} t_{2},
\end{array}
$$

in which all the letters with suffixes denote arbitrary integers. Since in the system (3) the two values of $x$ must be equal, we have

$$
r_{1} s_{1}^{2}=r_{2}^{2} s_{2}^{2}
$$

of which the complete integer solution is

$$
r_{1}=h f^{2}, \quad s_{1}=k g, \quad r_{2}=h g^{2}, \quad s_{2}=k f,
$$

where $f, g, h, k$ are arbitrary integers. From the (restricted) values $u \equiv a x+b, v \equiv c x+d$ of $u$, $v$, we find the stated conditions on $b, d$, after the change of notation $\left(t_{1}, t_{2}\right) \rightarrow(m, n)$. The completeness of the solutions of (3), (4) implies the completeness of the solution of (1) when $f, g, \cdots$ run through all solutions of (2). This treatment is possible only because (3), equivalent to (1), is a pure multiplicative system. A full discussion of such systems, with a non-tentative method for finding their complete solutions, was given in a former paper. ${ }^{3}$

The above solutions of (3), (4) were obtained by this method.

Proceeding similarly with

$$
a x^{2}+b x=y^{2}, \quad c x^{2}+d x=z^{2}, \quad e x^{2}+j x=w^{2},
$$

in which $a, b, c, d, e, j$ are constant integers, different from zero (not an essential restriction), we find that all solutions $x, y, z, w$ are given by

$$
\begin{array}{ll}
x=h f^{2} g^{2} k^{2} p^{2} q^{2} r^{2} s^{2}, & y=h f^{2} g k p q^{2} r s^{2} l, \\
z=h f^{2} g^{2} k^{2} p q r s m, & w=h f g^{2} k p q^{2} r^{2} s n,
\end{array}
$$

where $h, \cdots, m$ run through all integer solutions of

$$
\begin{gathered}
b=h f^{2} q^{2} s^{2}\left(l^{2}-a k^{2} g^{2} p^{2} r^{2}\right), \quad d=h f^{2} g^{2} k^{2}\left(m^{2}-c p^{2} q^{2} r^{2} s^{2}\right), \\
j=h g^{2} q^{2} r^{2}\left(n^{2}-e k^{2} f^{2} p^{2} s^{2}\right) .
\end{gathered}
$$

Here the simultaneous representation of $b, d, j$ which is necessary and sufficient for the existence of a solution of (5) is by forms of degree fifteen. This may suggest that anything approaching a complete solution of the systems quoted ${ }^{2}$ is not to be expected in the near future. However, any number of solvable systems can be constructed immedi-

${ }^{3}$ American Journal of Mathematics, vol. 55 (1933), pp. 50-66. 
ately from the conditions, and likewise for such systems having infinities of solutions.

The pure multiplicative system corresponding to (3) for (5) is

$$
r_{1} s_{1}^{2}=r_{2} s_{2}^{2}=r_{3}^{2} s_{3}^{2}
$$

of which the complete solution is

$$
\begin{array}{lll}
r_{1}=h f^{2} q^{2} s^{2}, & r_{2}=h f^{2} g^{2} k^{2}, & r_{3}=h g^{2} q^{2} r^{2}, \\
s_{1}=k g p r, & s_{2}=p q r s, & s_{3}=k f p s .
\end{array}
$$

The special features of (1), (5) and the systems cited ${ }^{2}$ which make it possible to treat the equations as a pure multiplicative system are that each equation lacks a constant term in one of its members and that its other member consists of a single term. The second of these restrictions is removed in $\$ 2$. It is immaterial that the single term has the coefficient unity; for multiplicative systems with arbitrary constant integer coefficients can also be solved completely by a straightforward, non-tentative process. ${ }^{3}$

For example, the pure multiplicative system corresponding to the system

$$
a x^{2}+b x=r y^{2}, \quad c x^{2}+d x=s z^{2},
$$

in which the constant integers $a, b, c, d, r, s$ are all different from zero, is

$$
x u=r y^{2}, \quad x v=s z^{2} .
$$

We first solve (7) as if all the letters were variable integers, and find the complete solution

$$
\begin{aligned}
& x=\operatorname{tr}_{1} r_{2} r_{4}^{2} s_{1} s_{2} s_{4}^{2} x_{1} x_{2}^{2} y_{1}^{2} y_{2}^{2}, \quad y=r_{2} s_{1} s_{2} s_{4} x_{1} x_{2} y_{1}^{2} y_{2} z_{1}, \\
& z=r_{1} r_{2} r_{4} s_{2} x_{1} x_{2} y_{1}^{2} y_{2} z_{2} \\
& u=r_{2} r_{3} s_{1} x_{1} y_{1}^{2} z_{1}^{2}, \quad v=r_{1} s_{2} s_{3} x_{1} y_{2}^{2} z_{2}^{2}, \\
& r=t r_{1} r_{2} r_{3} r_{4}^{2}, \quad s=t s_{1} s_{2} s_{3} s_{4}^{2},
\end{aligned}
$$

in which $t$ and all the letters with suffixes denote arbitrary integers. If now $r, s$ are constant, we resolve them in all possible ways into products of five factors as indicated above, and it suffices (as appears in the method $\operatorname{cited}^{3}$ ) to take $t$ equal to the G.C.D. of $r, s$. With each such pair of resolutions of $r, s$ there is associated a solution $x, y, z, u, v$ as given in (8); all the solutions of (7), with $r, s$ constant, fall into sets 
determined by these pairs. Proceeding as in (1)-(4), we find that (6) has an integer solution $x, y, z$ if and only if integers $x_{1}, x_{2}, y_{1}, y_{2}, z_{1}, z_{2}$ exist such that, for some resolution of $r, s$ as in (8),

$$
\begin{aligned}
& b=r_{2} s_{1} x_{1} y_{1}^{2}\left(r_{3} z_{1}^{2}-a t r_{1} r_{2} r_{4}^{2} s_{2}^{2} s_{4}^{2} x_{2}^{2} y_{2}^{2}\right), \\
& d=r_{1} s_{2} x_{1} y_{2}^{2}\left(s_{3} z_{2}^{2}-c t r_{2}^{2} r_{4}^{2} s_{1} s_{2} s_{4}^{2} x_{2}^{2} y_{1}^{2}\right) ;
\end{aligned}
$$

and if these conditions are satisfied, the complete solution $x, y, z$ of (7) is as given in (8), in which $t$ and the $r_{i}, s_{i}$ refer to all resolutions of the constants $r, s$ of the type indicated. The extension to a system of $n(>2)$ quadratics is immediate.

2. The general case. The notation is as follows.

$P_{i}(x)$ is a polynomial in $x$ of degree $m_{i}+r_{i}, m_{i}>0, r_{i}>0$, with constant integer coefficients.

The term of lowest degree in $P_{i}(x)$ is $a_{i} x^{m_{i}}, a_{i} \neq 0$.

$$
P_{i}(x) \equiv x^{m_{i}} Q_{i}(x), \quad Q_{i}(x) \equiv R_{i}(x)+a_{i},
$$

so that $R_{i}(x)$ is a polynomial in $x$ of degree $r_{i}>0$ with constant integer coefficients and no constant term.

$b_{i}, n_{i}, s$ denote constant integers, $b_{i} \neq 0, n_{i}>0, s>1$.

By the method exemplified in $\$ 1$, we may obtain necessary and sufficient conditions that the system

$$
P_{i}(x)=b_{i} y_{i}^{n_{i}}, \quad i=1, \cdots, s,
$$

shall have a solution in integers $x, y_{1}, \cdots, y_{s}$. The conditions concern the representability of the constant integers $a_{1}, \cdots, a_{s}$ in certain forms; the complete solution of (9) is known when all these representations of $a_{1}, \cdots, a_{s}$ are known.

Rewriting (9) as

$$
x^{m_{i}}\left[R_{i}(x)+a_{i}\right]=b_{i} y_{i}^{n_{i}}, \quad i=1, \cdots, s,
$$

we associate with (10) the pure multiplicative system

$$
x^{m_{i} z_{i}}=b_{i} y^{n_{i}}, \quad i=1, \cdots, s .
$$

The complete integer solution $x, z_{i}, y_{i}, i=1, \cdots, s$, of (11) is found as in $\S 1$ in terms of $t$, $\equiv t\left(m_{1}, \cdots, m_{s}, n_{1}, \cdots, n_{s}\right)$, parameters; say the solution is

$$
\begin{array}{r}
x=f\left(u_{1}, \cdots, u_{t}\right), \quad z_{i}=g_{i}\left(u_{1}, \cdots, u_{t}\right), \quad y_{i}=h_{i}\left(u_{1}, \cdots, u_{t}\right), \\
i=1, \cdots, s .
\end{array}
$$


The $f, g_{i}, h_{i}$ are power products in $u_{1}, \cdots, u_{t}$ with integer coefficients which are power products formed from the factors in certain resolutions of the constants $b_{1}, \cdots, b_{s}$ into products; the forms of $f, g_{i}, h_{i}$ and the coefficients described depend only on the given constant integers $m_{i}, n_{i}$. Necessary and sufficient conditions that the system (9) have a solution in integers $x, y_{i}, i=1, \cdots, s$, are found as in $\$ 1$, and refer to the representation of the constants $a_{i}$ in certain forms. Comparing (10), (11), we find

$$
a_{i}=g_{i}\left(u_{1}, \cdots, u_{t}\right)-R_{i}\left(f\left(u_{1}, \cdots, u_{t}\right)\right), \quad u=1, \cdots, s,
$$

as the required conditions. When these are satisfied, the solutions are obtained from (12).

An obvious modification gives necessary and sufficient conditions for the existence of an integer solution, and so on, of the more general system

$$
c_{i} P_{i}(x)=b_{i} T_{i}\left(y_{i}\right), \quad i=1, \cdots, s,
$$

in which $c_{i}, b_{i}$ are constants, $P_{i}(x)$ is as before, and $T_{i}\left(y_{i}\right)$ is a polynomial in $y_{i}$ of degree $n_{i}+t_{i}, n_{i}>0, t_{i}>0$, in which the term of lowest degree is $d_{i} y_{i}^{t_{i}}, d_{i} \neq 0$. The associated pure multiplicative system is

$$
c_{i} x^{m_{i}} z_{i}=b_{i} y_{i}^{n_{i}} w_{i}, \quad i=1, \cdots, s ;
$$

the conditions concern the representability of the $a_{i}, d_{i}$ in certain forms. As in all cases the conditions are both necessary and sufficient, the complete solution of the given system is equivalent to finding the total representation of the coefficients $a_{i}, d_{i}$ in a determinable system of forms.

California Institute of Technology 